\title{
Synthesis of Tungsten Oxide Nanorod, Its Application on Textile Material, and Study of Its Functional Properties
}

\author{
Abdul Azeem, ${ }^{1}$ Munir Ashraf, ${ }^{2}$ Usman Munir, ${ }^{1}$ \\ Zahid Sarwar, ${ }^{1}$ Sharjeel Abid, ${ }^{1}$ and Naeem Iqbal ${ }^{3}$ \\ ${ }^{1}$ Department of Postgraduate Studies, National Textile University, Faisalabad, Pakistan \\ ${ }^{2}$ Department of Textile Processing, National Textile University, Faisalabad, Pakistan \\ ${ }^{3}$ Health Biotechnology Division, National Institute for Biotechnology and Genetic Engineering, Faisalabad, Pakistan \\ Correspondence should be addressed to Abdul Azeem; abdulazeem88@hotmail.com
}

Received 11 June 2016; Revised 6 October 2016; Accepted 13 October 2016

Academic Editor: Oded Millo

Copyright (C) 2016 Abdul Azeem et al. This is an open access article distributed under the Creative Commons Attribution License, which permits unrestricted use, distribution, and reproduction in any medium, provided the original work is properly cited.

\begin{abstract}
Nanomaterial and its application in textiles are emerging as vast and diverse field due to enhanced functionalized characteristics. This study emphasizes the fabrication of tungsten trioxide nanostructured rods and analyzes its electrostatic and ultraviolet resistance properties. These nanorods are synthesized by hydrothermal method. Through hydrothermal method rod like nanostructures were grown on polyester fabric as it withstands curing temperature easily. The growth mechanism of the film is investigated. Electrostatic analysis of treated polyester fabric was failed but the analysis of seeded solution revealed that it has tunable transmittance modulation under different voltages and repetitive cyclic between the clear and blue states. Ultraviolet resistance of $100 \%$ seeded polyester fabric was higher than untreated fabric with respect to increasing concentration of nanorods. Results show that although the seeded solution is perfect, the conductivity of tungsten trioxide cannot be achieved on textiles.
\end{abstract}

\section{Introduction}

Tungsten oxide has gained much attention in recent years for its electrochromic, photo catalytic, photoluminescent, and gas sensing properties $[1,2]$. Electrochromic property, of all these characteristics, is the most highlighted as $\mathrm{WO}_{3}$ film has the ability to undergo optical coloration when voltage is applied. Further its nanoapplication in textile field is emerging for which this study has been designed $[3,4]$. The textiles can be categorized into two major types, conventional textiles and functional textiles. Conventional textiles include the items of general clothing and home textiles, while the functional textiles have their properties enhanced and are used for various functions and could be achieved more perfectly by application of nanomaterials. Over the years, studies have been made and researches are done to study numerous interactions of textiles with chemicals. Functional textiles have to play a multidimensional role; therefore they can also be called multifunctional textiles, for example, waterproof breathable fabrics, color changing textiles, and flame retardant, cut resistant, antibacterial fabrics [5].
Electrochromism is the phenomenon presented by some specific materials or compounds and it can be defined as the persistent but reversible change in color of specific materials or compounds when a burst of electricity is passed through them. These compounds undergo an electrochemical redox reaction. These optical properties are reversible; that is, the primary state is recoverable when the polarity of voltage is altered [6]. The principle of electrochromicity is as follows: this process allows specific materials or compounds to change their color or even opacity on the application of specific voltage and once required color or opacity has been achieved; no further electricity is needed. Electrochromic materials have crystalline or amorphous regions in their structure and mostly these have porous structures resulting in a swift ionic diffusion through open structure [7]. An amorphous $(\alpha)$ $\mathrm{WO}_{3}$ film has a definite ionic and electronic conduction. It has large opened porous structure and it is constituted by clusters. The clusters are built from $\mathrm{WO}_{6}$-octahedra, linked together by corners or edges and in the complete structure of the film connected with one another by W-O-W bonds or water bridges [8]. Among transition metal oxides tungsten 


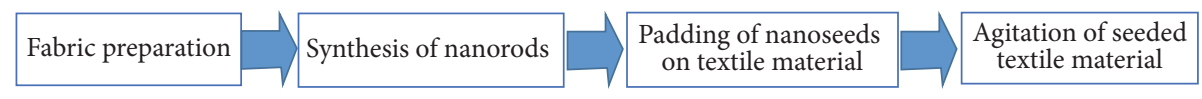

Figure 1

oxide $\left(\mathrm{WO}_{3}\right)$ has fast response time and high coloration, which is the reason of its extensive study. It has the property of changing its color from yellow to opaque blue when voltage is applied. For this purpose, high surface area of $\mathrm{WO}_{3}$ is required which could be obtained by production of thin $\mathrm{WO}_{3}$ with nanostructured particles [3, 4].

Previously Jiao and his companions worked on hydrothermally grown nanostructured $\mathrm{WO}_{3}$ films and found their application on glass plate as electrochromic material. Their work was initially based on inorganic electrochromic material. To gain the property of electrochromicity, they preferred the use of tungsten oxide $\mathrm{WO}_{3}$ as electrochromic material. They found that tungsten oxide shows blue color and becomes colorless by alternating the voltage applied polarity. Tungsten oxide parades a cyclic permanency and a high coloration efficiency as compared to other transition metal oxides. They discovered various methods for manufacturing the $\mathrm{WO}_{3}$ thin film electrodes. These methods were sputtering, pulsed laser deposition, electrosynthesis, sol gel, and hydrothermal method. Amongst them they filtered out hydrothermal method due to excellent results of produced particles. They found that electrosynthesis needs a high energy input and sol gel process followed by dip coating method requires bulky areas for deposition of $\mathrm{WO}_{3}$ films with high efficiency while hydrothermal method requires less temperature, springy substrate, and easiness in scaling up [9].

This study emphasizes the fabrication of tungsten trioxide nanorods grown on polyester fabric for enhanced electrostatic, electrochromic, and ultraviolet resistance properties in tungsten oxide treated textile substances.

\section{Experimental}

2.1. Materials. The fabric used in this work was polyester plain woven for easy growth of nanostructured materials. The fabric must be prepared before the application. In this work hydrochloric acid is used to make tungsten oxide precipitates [10]. Hydrogen peroxide $\left(\mathrm{H}_{2} \mathrm{O}_{2}\right)$ is simplest peroxide used for dissolving tungsten oxide precipitates to make solution. Sodium tungstate is a sodium salt of orthotungstate. It is inorganic in nature and has chemical formula of $\mathrm{Na}_{2} \mathrm{WO}_{3}$ used as the starting material for formation of tungsten oxide nanorods. $\mathrm{NaOH}$ was used for generating the polar sites over polyester fabric. Two-roll padder and hot air oven were used.

\subsection{Methods. The process flowchart is given in Figure 1.}

2.2.1. Fabric Preparation. The preparation process involves treatment of polyester fabric with $4 \%$ solution of $\mathrm{NaOH}$ at $90^{\circ} \mathrm{C}$ for 30 minutes by exhaust method. After this process the sample was extensively washed with water to remove all of the alkali present. The alkali treatment peels off the upper most surface of the polyester fiber and reveals the fresh surface with polar groups generated. The polar groups are necessary for the development of growth sites for incoming nanostructured materials on the fabric. Nanorods will not grow during agitation process without the growth sites. Smooth and lustrous fabric surface was obtained after whole process. The sample was oven-dried to remove moisture.

2.2.2. Synthesis of Nanorods. Required amount of sodium tungstate was taken and dissolved in freshly prepared distilled water. The solution was mechanically stirred until all of the sodium tungstate has been dissolved in distilled water. $\mathrm{HCl}$ was gradually added in the above prepared solution. Acid was added to that point where the further precipitation stops and a little extra may be added to make sure that the reaction has been completed. By adding $\mathrm{HCl}$, solution changes its color from crystal white to yellow and becomes viscous. The solution then filtered to remove excess of $\mathrm{HCl}$ and filtered precipitates are oven dried. The nanorods are now synthesized.

2.2.3. Padding of Nanoseeds on Textile Material. The ovendried precipitates were dissolved in $\mathrm{H}_{2} \mathrm{O}_{2}$ so that they can be applied over fabric. To make a solution of tungsten oxide precipitates, precipitates dissolved in specific amount of hydrogen peroxide with continuous stirring. While stirring, bubbling is avoided and a yellowish paste is obtained. This yellowish solution exhibits itself as seeds of tungsten oxide nanorods. The textile material, that is, polyester in this case, padded on two-roll padder with the solution, dried $\left(110^{\circ} \mathrm{C}\right.$, two minutes), and cured $\left(160^{\circ} \mathrm{C}\right.$, three minutes) on stentor frame. The whole step repeated five times; this was to ensure that seeds generated evenly all over the fabric. These seeds act as roots for the nanorods to grow. After the curing has been completed, the fabric was ready to be agitated.

2.2.4. Agitation of Seeded Textile Material. A small specimen of seeded textile material was taken in the round bottom flask and $\mathrm{H}_{2} \mathrm{O}_{2}$ solution containing dissolved nanoparticles of sodium tungstate. The flask was filled with as much solution as required to completely sink the specimen. An agitator capsule was also placed inside the flask. This plastic capsule, with magnet inside it, stirs the solution when placed on magnetic stirrer. A condenser was attached on top of the flask to stop the $\mathrm{H}_{2} \mathrm{O}_{2}$ from evaporating during agitation. The round bottom flask with the condenser attached was placed in water bath over hot plate magnetic stirrer. The temperature was set at $65^{\circ} \mathrm{C}$ and stirring at medium and agitation process was carried out for 6 hours. The specimen was then taken out 
TABLE 1: Performances with respect to preparing seeding solution.

\begin{tabular}{lcccc}
\hline Performances & $\mathrm{Na}_{2} \mathrm{WO}_{4} \cdot 2 \mathrm{H}_{2} \mathrm{O}$ (sodium tungstate) & Distilled water & $\mathrm{HCl}$ & $\mathrm{H}_{2} \mathrm{O}_{2}$ (hydrogen peroxide) \\
\hline 1 & 13.3 grams & $200 \mathrm{~mL}$ & Added until precipitations & $50 \mathrm{~mL}$ \\
2 & 13.3 grams & $200 \mathrm{~mL}$ & Added until precipitations & $50 \mathrm{~mL}$ \\
3 & 26.6 grams & $200 \mathrm{~mL}$ & Added until precipitations & $50 \mathrm{~mL}$ \\
4 & 26.6 grams & $200 \mathrm{~mL}$ & Added until precipitations & Added until clear solution \\
5 & 26.6 grams & $100 \mathrm{~mL}$ & Added until precipitations & Added until clear solution \\
6 & 26.6 grams & $100 \mathrm{~mL}$ & Added until precipitations & Used $5 \%$ seeds \\
7 & 26.6 grams & $100 \mathrm{~mL}$ & Added until precipitations & Used $15 \%$ seeds \\
8 & 26.6 grams & $100 \mathrm{~mL}$ & Added until precipitations & Used $30 \%$ seeds \\
9 & 26.6 grams & $100 \mathrm{~mL}$ & Added until precipitations & Used $50 \%$ seeds \\
10 & 26.6 grams & $100 \mathrm{~mL}$ & Added until precipitations & Used $75 \%$ seeds \\
11 & 26.6 grams & $100 \mathrm{~mL}$ & Added until precipitations & Used $100 \%$ seeds \\
12 & 40 grams & $100 \mathrm{~mL}$ & Added until precipitations & Added until clear solution \\
13 & 20 grams & $50 \mathrm{~mL}$ & & 4 mL \\
\hline
\end{tabular}

TABle 2: Performances with respect to caustic treatment and curing conditions.

\begin{tabular}{|c|c|c|c|c|c|}
\hline \multirow{2}{*}{ Performances } & \multicolumn{3}{|c|}{ Caustic treatment } & \multicolumn{2}{|c|}{ Curing } \\
\hline & $\mathrm{NaOH}$ & Temperature & Time & Temperature & Time \\
\hline 1 & \multicolumn{5}{|c|}{ Cotton fabric was damaged } \\
\hline $2-11$ & $40 \mathrm{~g} / \mathrm{L}$ & $90^{\circ} \mathrm{C}$ & $30 \mathrm{~min}$ & $180^{\circ} \mathrm{C}$ & $3 \mathrm{~min}$ \\
\hline 12 & $40 \mathrm{~g} / \mathrm{L}$ & $90^{\circ} \mathrm{C}$ & $30 \mathrm{~min}$ & $165^{\circ} \mathrm{C}$ & $3 \mathrm{~min}$ \\
\hline 13 & $40 \mathrm{~g} / \mathrm{L}$ & $90^{\circ} \mathrm{C}$ & $30 \mathrm{~min}$ & $150^{\circ} \mathrm{C}$ & $3 \mathrm{~min}$ \\
\hline
\end{tabular}

with the help of tweezers (avoid hand contact), rinsed once with water, and oven-dried.

2.3. Performances (Design of Experiments). Table 1 represents the different performances with respect to making seeded solution carried out for this work. A fabric sample of $2 \times 1$ inches was taken in all performances; cotton fabric in first and polyester in all other performances were taken.

Table 2 represents the different performances with respect to caustic treatment and growth of seeded solution onto the fabrics at different conditions carried out for this work. A fabric sample of $2 \times 1$ inches was taken in all performances; cotton in first and polyester in all other performances were taken.

\subsection{Methods of Performances}

2.4.1. Performance 1. $200 \mathrm{~mL}$ distilled water was poured into a beaker. 13.3-gram $\mathrm{Na}_{2} \mathrm{WO}_{4} \cdot 2 \mathrm{H}_{2} \mathrm{O}$ (sodium tungstate) was weighed on weight balance, added in beaker containing distilled water, and mixed thoroughly until a clear solution appears. Hydrochloric acid ( $\mathrm{HCl})$ was added in the solution which yields yellow precipitates; $\mathrm{HCl}$ was added in excess to complete the reaction (all of the dissolved sodium tungstate particles precipitate out). The precipitates were then filtered with the help of filter paper. After filtration the precipitates were dried on the filter paper inside the oven such that the filter paper was not damaged due to $\mathrm{HCl}$. The dried precipitates were then separated in a dish and saved. $50 \mathrm{~mL}$ hydrogen peroxide $\left(\mathrm{H}_{2} \mathrm{O}_{2}\right)$ was taken in a beaker and the saved precipitates were added in it to make a seed solution. Mercerized cotton fabric of dimensions $2 \times 1$ inches was taken and padded after dipping in the seed solution and then dried in the oven. Due to the presence of acid the cotton fabric was damaged so further procedure was unable to proceed.

2.4.2. Performance 2. Seed solution was prepared according to the performance one. $100 \%$ polyester of dimensions $2 \times$ 1 inches was taken as polyester is acid friendly. Polyester does not have any sites of attachment of nanoseeds; the sites were first created by caustic treatment. $40 \mathrm{~g} / \mathrm{L} \mathrm{NaOH}$ was taken in a beaker and the given polyester fabric was added in it. The beaker was then put into a water bath and treated for 30 minutes at $90^{\circ} \mathrm{C}$. After 30 minutes the fabric was washed thoroughly to make sure all the $\mathrm{NaOH}$ was removed from the fabric structure, and then it was oven-dried. The caustic treated polyester was then dipped in the seed solution, prepared earlier, padded on the padder, and then dried in the oven at $100^{\circ} \mathrm{C}$ for two minutes. The dip-pad-dry procedure was repeated five times. After fifth drying, the fabric was cured over stentor frame at $180^{\circ} \mathrm{C}$ for 3 minutes. Then the seed solution was poured into a round bottom flask and placed on a magnetic stirrer in a water bath at a temperature of $60^{\circ} \mathrm{C}$. The cured fabric was added into the seed solution in round bottom flask, an agitator was dropped in the flask, and a condenser was adjusted on its top with the connections of cold water. The fabric was stirred in the flask with the help of agitator alongside heating at $60^{\circ} \mathrm{C}$. The process was run 
for 6 hours by checking at regular intervals that the fabric agitates thoroughly. After 6 hours the treated polyester was dried at $100^{\circ} \mathrm{C}$ and then cured at $160^{\circ} \mathrm{C}$ for 5 minutes; drying and curing were done over stentor frame.

2.4.3. Performance 3. Sites on polyester fabric were created as the same as mentioned in performance two. Seed solution was prepared according to the same procedure in performance one, except that the quantity of sodium tungstate dissolved in distilled water was doubled. The further procedure of fabric dip-pad-dry was carried out the same way as mentioned earlier and then it was cured. Agitation procedure was done as discussed in performance two and then the fabric was dried and cured.

2.4.4. Performance 4. Sites on polyester fabric were created by the same method of caustic treatment as in performance two, with fabric washed and dried. For making the seed solution, the procedure was repeated as in performance one but the difference was that the $\mathrm{H}_{2} \mathrm{O}_{2}$ was added until a clear solution was formed. The rest of the procedure was same but, during dip-pad-dry process, when dipped for third time the solution bubbled up and when put in the round bottom flask again the whole system bubbled and the procedure could not be carried out further.

2.4.5. Performance 5. Sites growth procedure on polyester was again the same as in performance two. For the preparation of seed solution, 26.6 gram $\mathrm{Na}_{2} \mathrm{WO}_{4} \cdot 2 \mathrm{H}_{2} \mathrm{O}$ (sodium tungstate) was added in $100 \mathrm{~mL}$ water and mixed thoroughly to form a clear solution and then concentrated $\mathrm{HCl}$ was added until all of the dissolved sodium tungstate was converted into precipitates. After the precipitates were formed the solution was washed by rinsing with water for 2-3 times to obtain acid-free solution in order to avoid bubbling during further performance. Then the precipitates were filtered and dried. Dried precipitates were taken into a beaker and $\mathrm{H}_{2} \mathrm{O}_{2}$ was added until a clear solution appears, that is, the seed solution. Then further methodology was repeated as in performance two.

2.4.6. Performance 6. Procedure for growth of sites on polyester was the same. Precipitates were made and dried according to the same method as discussed in performance five. After drying, the seeds were saved in a closed dish. Then the seed solution was prepared with $5 \%$ seeds in $\mathrm{H}_{2} \mathrm{O}_{2}$ solution. The rest of procedure was repeated as discussed earlier in previous performances.

2.4.7. Performance 7. Whole procedure was repeated according to the same method as in performance 6 . The seed solution was created by adding $15 \%$ seeds in $\mathrm{H}_{2} \mathrm{O}_{2}$ solution. The rest of procedure was repeated as discussed earlier in previous performances.

2.4.8. Performance 8. Whole procedure was repeated according to the same method as in performance 6 . The seed solution was created by adding $30 \%$ seeds in $\mathrm{H}_{2} \mathrm{O}_{2}$ solution.
The rest of procedure was repeated as discussed earlier in previous performances.

2.4.9. Performance 9. Whole procedure was repeated according to the same method as in performance 6 . The seed solution was created by adding $50 \%$ seeds in $\mathrm{H}_{2} \mathrm{O}_{2}$ solution. The rest of procedure was repeated as discussed earlier in previous performances.

2.4.10. Performance 10. Whole procedure was repeated according to the same method as in performance 6 . The seed solution was created by adding $75 \%$ seeds in $\mathrm{H}_{2} \mathrm{O}_{2}$ solution. The rest of procedure was repeated as discussed earlier in previous performances.

2.4.11. Performance 11. Whole procedure was repeated according to the same method as in performance 6 . The seed solution was created by adding $100 \%$ seeds in $\mathrm{H}_{2} \mathrm{O}_{2}$ solution. The rest of procedure was the same.

2.4.12. Performance 12. $100 \%$ polyester was treated with $40 \mathrm{~g} / \mathrm{L}$ caustic $(\mathrm{NaOH})$ solution to create sites for reaction $100 \mathrm{~mL}$ water that was taken and 40 -gram $\mathrm{Na}_{2} \mathrm{WO}_{4} \cdot 2 \mathrm{H}_{2} \mathrm{O}$ (sodium tungstate) was added in it, mixed thoroughly to make a clear solution. Then concentrated $\mathrm{HCl}$ was added until all the particles were created to yellow precipitates. Then the precipitates were filtered with the help of filter paper and then dried. The filtered precipitates were then washed by rinsing in water 2-3 times and then again filtered and dried. The dried particles were then added with $\mathrm{H}_{2} \mathrm{O}_{2}$ until a clear solution appeared. Then treated polyester was dip-paddried for 5 times in the solution with the help of padder and then cured in stentor frame at $180^{\circ} \mathrm{C}$ for three minutes. The cured fabric was then operated for almost six hours in the round bottom flask arrangement discussed in performance two. After six hours the fabric was dried and then cured, making sure that the fabric does not come in contact with the skin or metal during the process.

2.4.13. Performance 13. Caustic treated polyester fabric was prepared according to the procedure in performance two. $20 \mathrm{~g}$ sodium tungstate was dissolved in $50 \mathrm{~mL}$ distilled water and clear solution was obtained. $4 \mathrm{~mL}$ of $\mathrm{HCl}$ was added to form the precipitates. In the same solution, $\mathrm{H}_{2} \mathrm{O}_{2}$ was gradually added to dissolve the precipitates formed. The solution formed was used to pad-dry-cure the caustic treated polyester fabric, measuring $2 \times 1$ inches, five times. Curing was done at $160^{\circ} \mathrm{C}$ for three minutes and drying at $100^{\circ} \mathrm{C}$ for two minutes over stentor frame. The fabric was then agitated with the seed solution for 4 hours according to the agitation procedure in performance two. The fabric was then dried and observed under scanning electron microscope.

\section{Testing Methods}

The tests performed on the samples prepared are discussed in detail in this section. The tests performed were UV resistance 


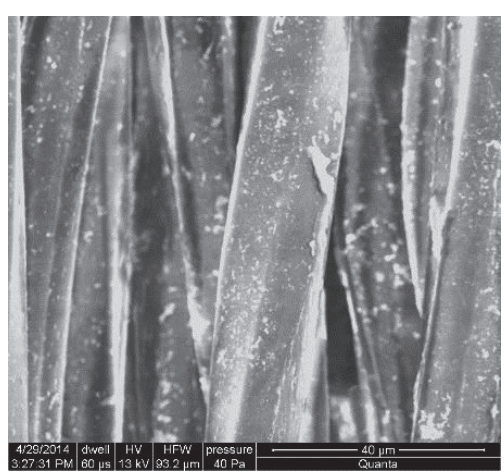

(a)

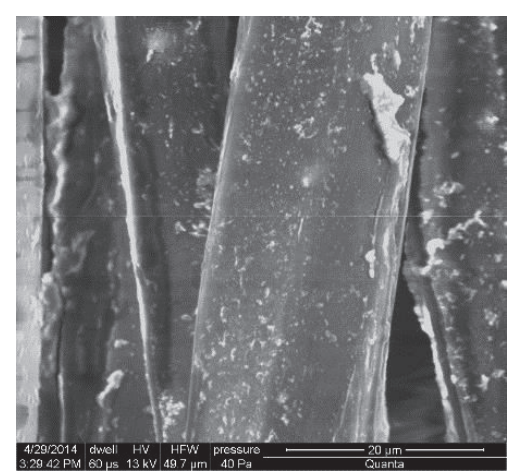

(b)

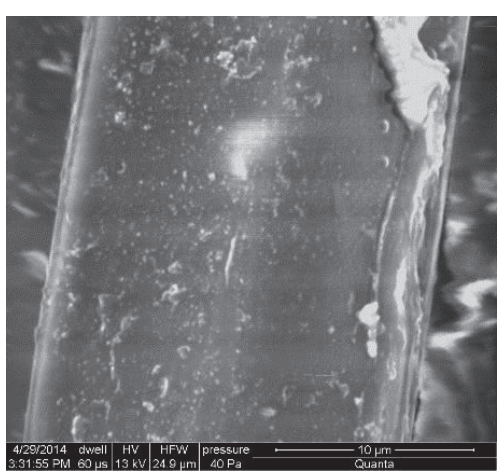

(c)

FIgURE 2: (a) Treated fabric at 1600x magnification. (b) Treated fabric at 3000x magnification. (c) Treated fabric at 6000x magnification.

tests, scanning electron microscope (SEM) analysis, and electrochromicity tests.

3.1. Electrochromicity. The samples prepared were checked for electrical conductivity and electrochromism by applying $3 \mathrm{~V} \mathrm{AC}$ and $-3 \mathrm{~V}$ AC; the voltage applied was gradually increased to $30 \mathrm{~V}$ AC and $-30 \mathrm{~V}$ AC. The voltage was applied by piercing the wires through the specimen. Seed solutions of different concentrations were prepared. The prepared solutions were $5 \%, 15 \%, 30 \%, 50 \%, 75 \%, 100 \%$, and $200 \%$ concentrations. These solutions were electrolyzed, starting from $3 \mathrm{~V}$ AC and gradually increasing the voltage to $15 \mathrm{~V}$ AC. The solutions above $50 \%$ concentration caused severe bubbling. However, the solutions below $50 \%$ concentrations changed their color to light blue and retained the shade even after the applied voltage was removed.

\subsection{Ultraviolet Resistance. Ultraviolet resistance test was} carried out for all of the saved treated fabric samples. This test on different treated fabric samples was carried out to check the ultraviolet resistance for each of the fabric samples and for comparison of ultraviolet resistivity for all of them.

\section{Results and Discussion}

4.1. SEM Analysis. Prepared specimens were analyzed under the scanning electron microscope. The scanning electron microscope results from the performance 2 are shown.

Figure 2(a) is the 1600 times magnified image of the polyester fabric specimen prepared in performance two. Each individual strand in the picture is the individual polyester fiber inside the polyester yarns of the fabric. A small amount of deposition can be seen everywhere on the fiber. These are the deposited nanoparticles. Figure 2(b) is the 3000 times magnified image of the polyester fabric specimen prepared in performance two. The image focusses on the same area of the fabric as in Figure 2(a). In the background (on both left and right sides) deposition of the nanoparticles can be seen in larger amount. In the foreground, a small amount of deposition is visible. Figure 2(c) is the 6000 times magnified image, focusing on the same area of the fabric as in
Figure 2(a). The figure shows accumulated nanorods on the surface of the polyester fiber.

4.1.1. Discussion on SEM Analysis. The SEM analysis tells us that nanorods are present on the fabric surface but they are not in the required concentration. They are accumulating on the specific sites of the fiber. This suggests that an external force has to be present in order to align these particles from head to toe. The external force can be an electric charge or magnetic field which can forcefully align these nanorods in the required position. The analysis also suggests that fibers have less sites for deposition. To increase the number of sites, there are two ways:

(i) Increase the time of caustic treatment and move the fabric continuously throughout the process. It will also allow the caustic soda $(\mathrm{NaOH})$ to interact more with the fabric surface and continuous movement will ensure the regularity of the surface.

(ii) Increase the concentration of caustic and move the fabric continuously throughout the process. Increase in concentration will also allow more molecules to act on the fabric surface.

4.1.2. Best Performance. Performance 13 was the performance that yielded the best results. The process was based on balancing the chemical equation of the reactions taking place and using the precise amount of chemical needed, neither more nor less.

Seeded Specimens. See Figure 3.

Treated Specimens. See Figure 4.

SEM images of pretreatment and posttreatment of substrate with nanorods are shown in Figures 3(a), 3(b), 3(c), 4(a), 4(b) and 4(c) respectively, which show that before treatment there are clear fiber surfaces shown with no nanoparticles and after the treatment the nanorods are visible all over the fiber in the form of three-dimensional structure. The electrochromism for this specimen did not yield any results. The possible reason is that even though the threedimensional structure has been formed over the polyester 


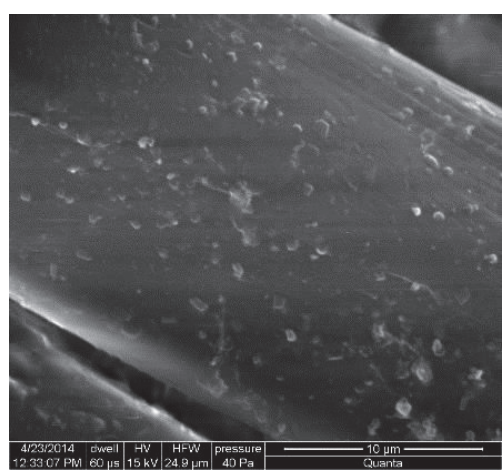

(a)

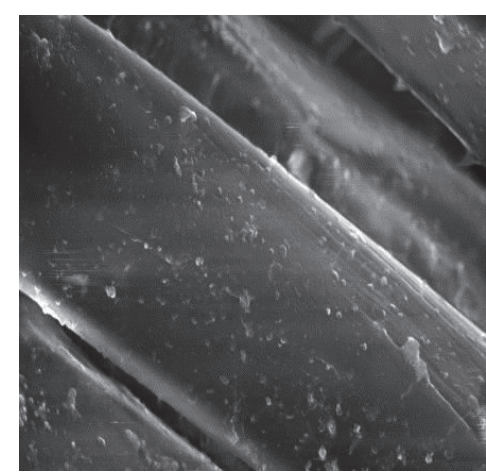

(b)

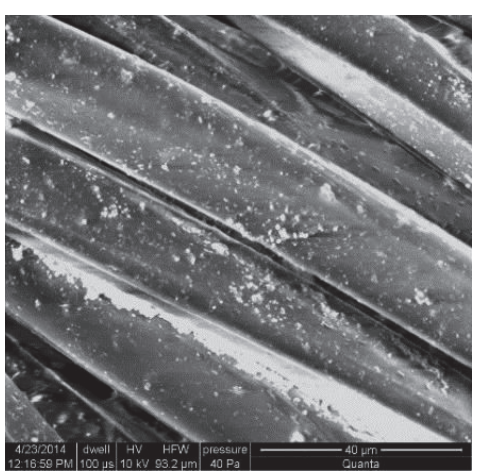

(c)

Figure 3: (a) Treated fabric at 6000x magnification. (b) Treated fabric at 3000x magnification. (c) Treated fabric at 1600x magnification.

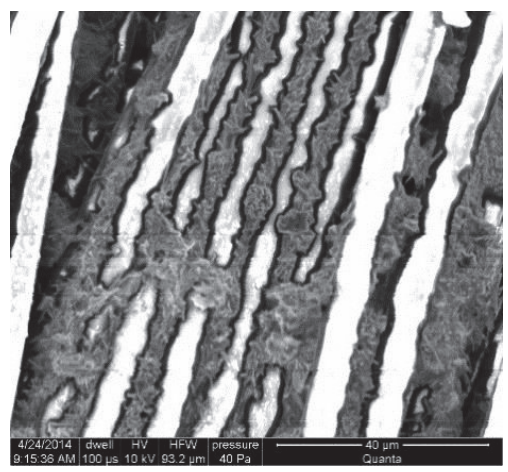

(a)

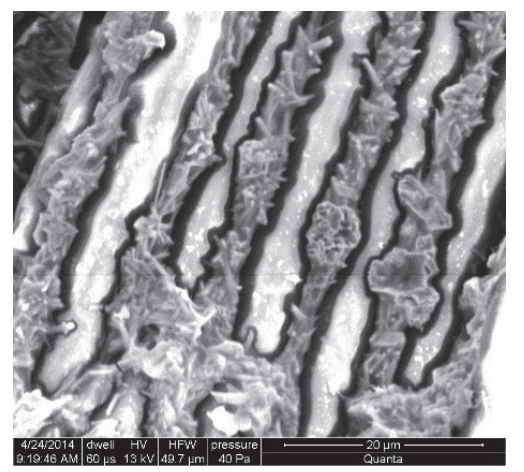

(b)

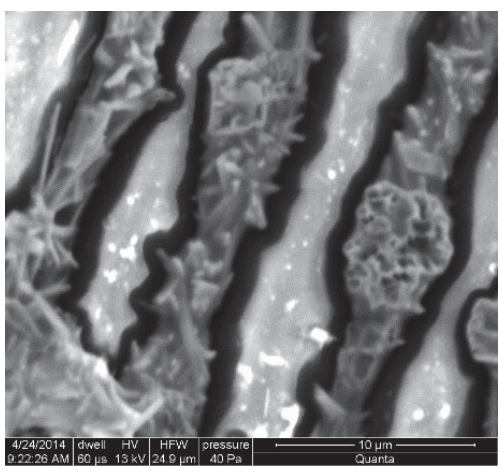

(c)

Figure 4: (a) Treated sample at 600x magnification. (b) Treated sample at 3000x magnification. (c) Treated sample at 6000x magnification.

fibers by the nanorods, they are not joined in head to tail manner to allow the electric current to pass through them. Some external force is required to align the nanorods as discussed earlier. Another observation was also seen; it may be an illusion but the fabric showed slight blue tint right after the curing process was complete.

4.2. Ultraviolet Resistance. The treated fabric specimens from performances 5 to 12 were checked for the ultraviolet resistance. The results mentioned in Table 3 were obtained.

Table 3 shows the UVA, UVB blockage in percentage, and mean ultraviolet protection factor.

According to the standards, the fabric with mean ultraviolet protection factor (UPF) $>40$ offers excellent protection against the ultraviolet rays. The pure polyester has the mean UPF of 27.2. As the concentration increases, the mean UPF also increases. The polyester fabric treated with the seed solution of $75 \%$ concentration offers excellent protection against the UV rays. Above $75 \%$ the mean UPF increases while, below $75 \%$, the mean UPF decreases. The UV resistance makes the treated polyester fabric ideal for the outdoor use. It will not be degraded and the fabric will protect what is beneath it from the UV rays. The above-mentioned results of UV blocking versus different samples have also been shown in Figure 5.
TABLE 3: UVA and UVB blocking percentage and mean UPF.

\begin{tabular}{lccc}
\hline Type & Mean UPF & UVA blocking & UVB blocking \\
\hline Un treated & 27.2 & $75.7 \%$ & $99.3 \%$ \\
$100 \%$ seeded & 58.1 & $87.7 \%$ & $99.5 \%$ \\
$100 \%$ sites & 24.1 & $75.1 \%$ & $99.0 \%$ \\
$200 \%$ seeded & 35.3 & $81.3 \%$ & $99.4 \%$ \\
$200 \%$ sites & 108.1 & $91.1 \%$ & $99.9 \%$ \\
$30 \%$ seeded & 27.3 & $77.4 \%$ & $99.2 \%$ \\
$30 \%$ sites & 37.1 & $81.5 \%$ & $99.4 \%$ \\
$75 \%$ seeded & 39.4 & $84.2 \%$ & $99.4 \%$ \\
$75 \%$ sites & 28.1 & $78.2 \%$ & $99.2 \%$ \\
$50 \%$ seeded & 31.4 & $78.6 \%$ & $99.4 \%$ \\
$50 \%$ sites & 60 & $87.9 \%$ & $99.6 \%$ \\
$5 \%$ seeded & 29.3 & $76.4 \%$ & $99.4 \%$ \\
$5 \%$ sites & 21.8 & $72.0 \%$ & $98.9 \%$ \\
\hline
\end{tabular}

In this table: untreated: pure polyester fabric without any process done on it. Sites: polyester fabric treated with seed solution (pad-dry-cure). Seeded: polyester fabric with the nanorods grown over it (fully treated).

\section{Conclusion}

The UV resistance property was achieved by each of the 13 samples. As the concentrations of seeded solution on 


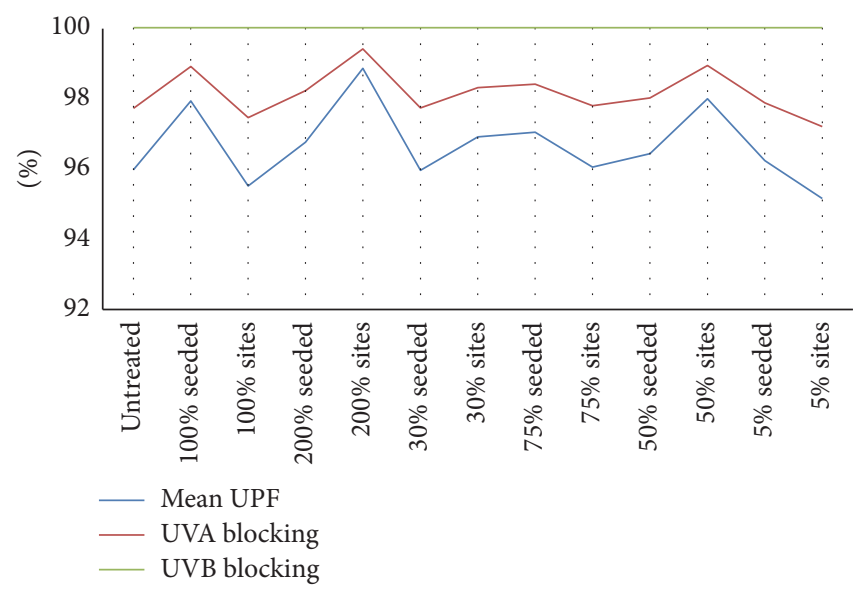

FIGURE 5: Graph of mean UPF, UVA, and UVB blocking percentages.

polyester fabric increase the mean UPF also increases. So it was concluded that these samples can be used for outdoor applications. Despite all the trails we were unable to achieve the property of electrochromicity on textile material which was polyester in our case. However, the as prepared solution showed property of electrochromicity and changed its color successfully on applying voltage. There was a problem in characterization of tungsten oxide nanorods due to which nanorods failed to show electrochromicity.

\section{Future Work}

Once the characterization has been successfully completed, the electrochromic fabric can have its application in various ways. It can be used to replace the high energy consuming neon sign boards. It can also be used as a flexible display. The only thing characterization now needs is the external force. As discussed earlier, this external element can be magnetic field or electric charge. It is not tested but applying electrical charge during agitation may align the nanorods in the required direction. We have already seen that, upon electrolysis, the seed solution changes its color from being colorless to light blue.

\section{Competing Interests}

The authors declare that they have no competing interests.

\section{References}

[1] Z. Zhou, B. Kong, C. Yu et al., "Tungsten oxide nanorods: an efficient nanoplatform for tumor CT imaging and photothermal therapy," Scientific Reports, vol. 4, article 3653, 2014.

[2] X. P. Wang, B. Q. Yang, H. X. Zhang, and P. X. Feng, “Tungsten oxide nanorods array and nanobundle prepared by using chemical vapor deposition technique," Nanoscale Research Letters, vol. 2, no. 8, pp. 405-409, 2007.

[3] R. Rattanakam and S. Supothina, "Hydrothermal synthesis and electrochromic properties of potassium tungsten oxide nanorods," Journal of Nanoscience and Nanotechnology, vol. 11, no. 10, pp. 8974-8978, 2011.

[4] H.-S. Shim, J. W. Kim, Y.-E. Sung, and W. B. Kim, "Electrochromic properties of tungsten oxide nanowires fabricated by electrospinning method," Solar Energy Materials and Solar Cells, vol. 93, no. 12, pp. 2062-2068, 2009.

[5] "Functional Textiles, Functional Apparel, Clean Room Textiles, Chemical Protective Clothing," http://www.fibre2fashion.com/ industry-article/11/1044/functional-textiles-and-apparels1.asp.

[6] "Innoshade: Principle of Electrochromism," http://www .innoshade.eu/principle-of-electrochromism.html.

[7] Liquid Crystals and Photonics Group, "Electrochromic displays," https://lcp.elis.ugent.be/tutorials/tut_echrom.

[8] J. A. Horsley, I. E. Wachs, J. M. Brown, G. H. Xia, and F. D. Hardcastle, "Structure of surface tungsten oxide species in the tungsten trioxide/alumina supported oxide system from x-ray absorption near-edge spectroscopy and Raman spectroscopy," The Journal of Physical Chemistry A, vol. 91, no. 15, pp. 40144020, 1987.

[9] Z. Jiao, X. W. Sun, J. Wang, L. Ke, and H. V. Demir, "Hydrothermally grown nanostructured $\mathrm{WO}_{3}$ films and their electrochromic characteristics," Journal of Physics D: Applied Physics, vol. 43, no. 28, Article ID 285501, 2010.

[10] "Standard Operating Procedure," https://chemistry.ucmerced .edu/sites/chemistry.ucmerced.edu/files/page/documents/ejm hydrochloric_acid.pdf. 

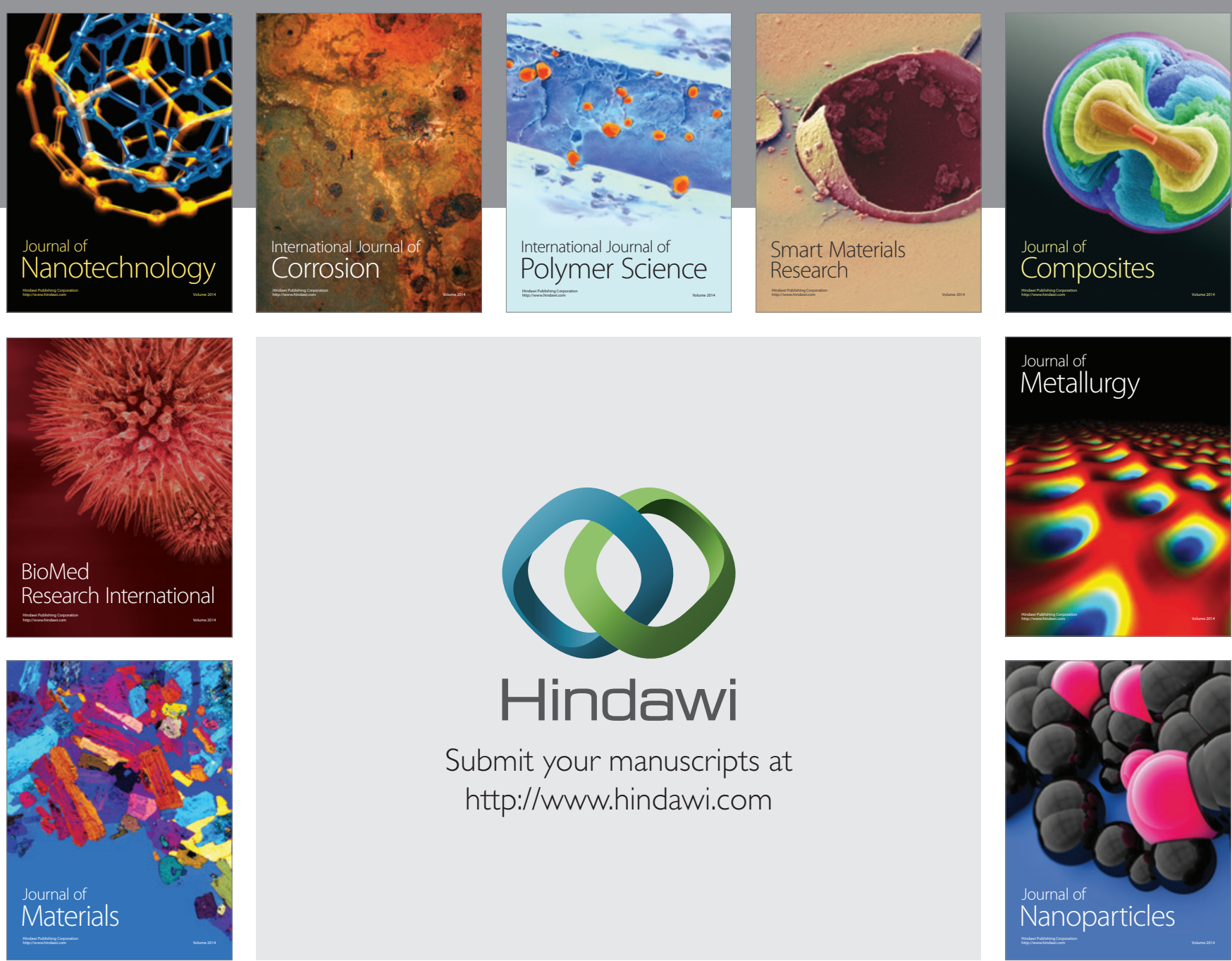

\section{Hindawi}

Submit your manuscripts at

http://www.hindawi.com

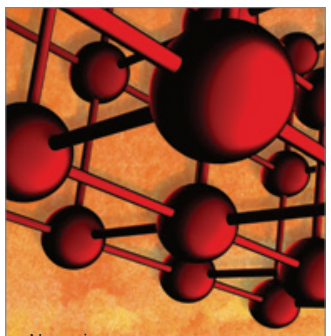

Materials Science and Engineering
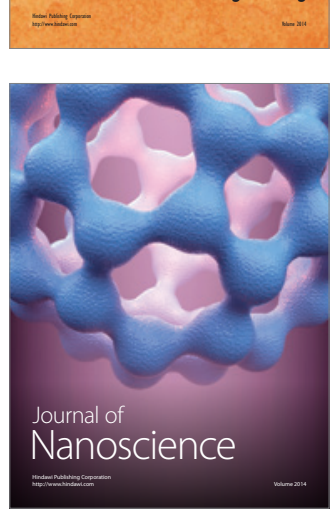
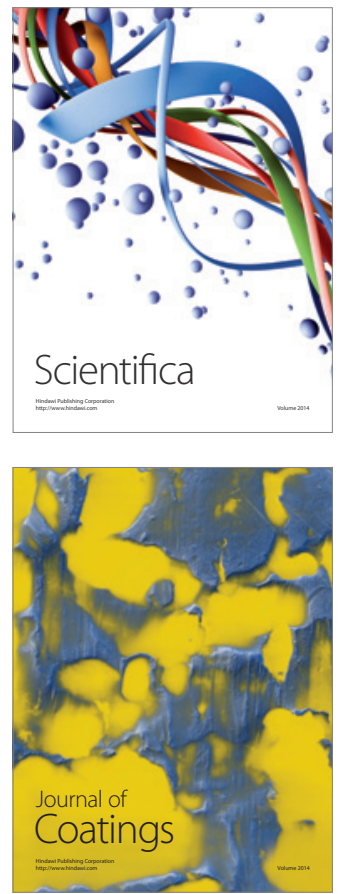
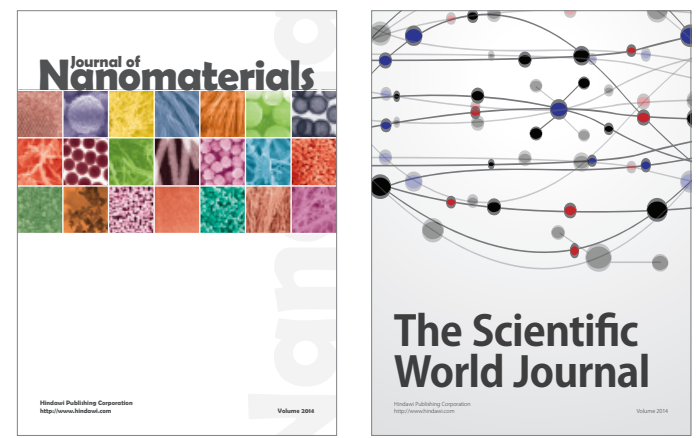

The Scientific World Journal
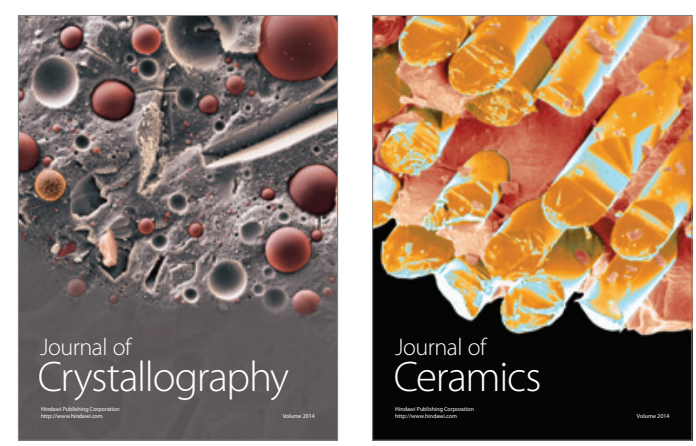
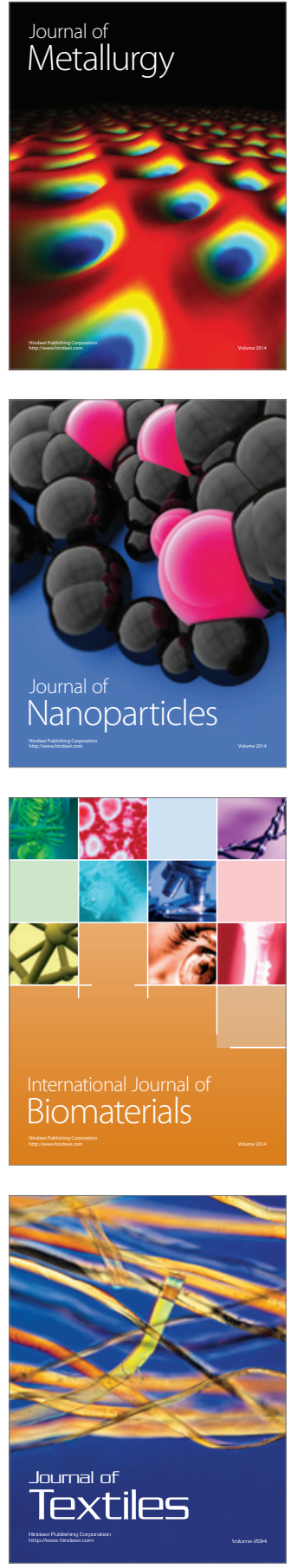